\title{
The Contribution of Texture Contrasts and Combinations to Food Acceptance Across Cultures
}

Authors: Robert Pellegrino ${ }^{1,6}$, Bobby Cheon ${ }^{2,3}$, Ciaran Forde ${ }^{2,4}$, Anna Oleszkiewicz ${ }^{5,6}$, Michal Pieniak $^{5}$, and Curtis Luckett ${ }^{1, *}$

${ }^{1}$ Department of Food Science, University of Tennessee, Knoxville, Tennessee

${ }^{2}$ Clinical Nutrition Research Centre (CNRC), Singapore Institute for Clinical Sciences, Agency for Science, Technology and Research (A*STAR), Singapore

${ }^{3}$ School of Social Sciences (Psychology), Nanyang Technological University, Singapore

${ }^{4}$ Department of Physiology, Yong Loo Lin School of Medicine, National University of Singapore, Singapore

${ }^{5}$ Institute of Psychology, University of Wroclaw, Wroclaw, Poland

${ }^{6}$ Smell \& Taste Clinic, Department of Otorhinolaryngology Technische Universität Dresden, Dresden, Germany

Corresponding author: Curtis R. Luckett; Department of Food Science, University of Tennessee, 2510 River Drive, Knoxville, TN 37996, U.S., cluckett@utk.edu

\begin{abstract}
Texture has long been considered an important attribute for food acceptance. However, which specific textural characteristics contribute to overall acceptance of a food is not well understood. It has been suggested that texture contrasts and combinations are a universal feature in giving foods a desirable texture, yet this notion is largely based upon anecdotal data. This study uses multiple survey research methods to assess the importance of texture contrast and combinations across cultures (Poland, U.S.A., and Singapore). Participants $(\mathrm{n}=288)$ completed a survey that included overt measures of food texture contrast importance as well as free response questions regarding texture. The overall importance of texture for food liking was not different across the populations. However, the participants from Singapore and Poland gave more importance to a desirable food having multiple textures than the U.S.A. cohort. When looking at free responses, participants were twice as likely to mention combinations (multiple textures) with a texture
\end{abstract}


contrast when describing foods they liked, in comparison to foods they disliked. This was observed across all 3 cultures. However, the type and quantity of texture terms used within combinations were different among cultures. For instance, Asians enjoyed more texturally diverse food combinations than the other two cultures. These findings highlight the importance of texture contrasts and combinations in three distinct cultures.

Keywords: texture; cross cultural; food preference 


\section{Introduction}

Texture is the sensation that arises from the combination of structural, mechanical, and surface properties of the foods detected through the senses of vision, hearing, and touch. In other words, texture is the way food and drink feels in one's mouth. Textures play an important role in food acceptance, with a majority of individuals giving it high importance in comparison to other characteristics of food (Luckett \& Seo, 2015; Szczesniak, 1971; Szczesniak \& Kahn, 1971). The importance of texture to palatability has been reported to be universal across populations (Rohm, 1990). Additionally, texture has been shown to be more important than flavor in the rejection of some foods for adults and children alike (Cardello, 1996; Szczesniak, 1972). For this reason, research interest in food texture has recently enjoyed a resurgence in academic and industry sectors.

Texture perception often begins with the eyes, but is centered on tactile sensations from within the oral cavity. The movement of the jaw and tongue, along with lubrication from saliva, manipulate the texture to make it safe for ingestion. Throughout the manipulation step, the texture of the food changes dynamically. Nomenclature for describing textural qualities was first developed in the 1960s (Szczeniak, 1963). The terms classified were designed to be simple in wording to relate fundamental, measurable properties with perceptual experience. Specifically, texture terms were classified into 3 main classes of characteristics and subsequent primary or secondary properties (see Table 1). Three main classes of characteristics were defined: mechanical, geometrical and other. Mechanical characteristics included texture properties that relate to applied stress (e.g. hardness, elasticity). Geometrical characteristics have properties related to structure and appearance (e.g. particle size and shape) while other characteristics included mouthfeel properties that do not fall into the first two categories (e.g. moisture and fat content). However, as mentioned by Szczesniak and later Guinard and Mazzuchelli (1996), some 
terms are more complex and may fit across categories or be context-specific like juiciness

(Guinard \& Mazzucchelli, 1996). Studies on consumer attitudes toward food attributes show that texture terms, such as crisp/crispy, crunch/crunchy, creamy and juicy, are very commonly reported by consumers (Szczesniak \& Kahn 1972; Luckett \& Seo 2015). Furthermore, in similar cross-cultural studies, overlap and differences in texture terms frequently used for foods among several populations have been shown (Lawless, Vanne, \& Tuorila, 1997; Nishinari et al., 2008; Rohm, 1990). A list for 54 English texture terms for foods and their equivalent term in 22 languages has also been compiled (Drake, 1989).

Table 1. Classification of textural characteristics by 3 main classes (mechanical, geometric, \& other) ${ }^{\mathrm{a}}$

\begin{tabular}{|c|c|c|}
\hline \multicolumn{3}{|l|}{ Mechanical characteristics } \\
\hline Primary & Secondary & Examples \\
\hline Hardness & & Soft, firm, hard \\
\hline \multirow[t]{3}{*}{ Cohesiveness } & Brittleness & Crumbly, crunchy, brittle \\
\hline & Chewiness & Tender, chewy, tough \\
\hline & Gumminess & Short, mealy, pasty, gummy \\
\hline Viscosity & & Thin, viscous \\
\hline Elasticity & & Plastic, elastic \\
\hline Adhesiveness & & Sticky, tacky, gooey \\
\hline \multicolumn{3}{|l|}{ Geometric characteristics } \\
\hline Primary & & Examples \\
\hline Particle size and shape & & Gritty, grainy, course \\
\hline Particle shape and orientation & & Fibrous, cellular, crystalline \\
\hline \multicolumn{3}{|l|}{ Other characteristics } \\
\hline Primary & Secondary & Examples \\
\hline Moisture content & & Dry, moist, wet, watery \\
\hline \multirow[t]{2}{*}{ Fat content } & Oiliness & Oily \\
\hline & Greasiness & Greasy \\
\hline
\end{tabular}

${ }^{a}$ Adapted from Szczeniak (1963) by Guinard \& Mazzuchelli (1996)

As mentioned, foods possess different texture types which manifest temporally during the eating process. So, while a food often contains multiple textures in a static state (i.e. chewy 
cheese and the crispy crust of pizza) multiple textures can be elicited dynamically during consumption, for example chocolate moving from a hard texture upon the first bite to a smooth texture prior to swallowing. A change in texture and other flavor properties has been discussed as a key for high palatability of food (Hyde \& Witherly, 1993). Different textures may also be classified depending on the energy required to process or manipulate them during consumption, and texture changes may move between two similar energy requirements or one having higher or lower (contrasting) energy needs than the other (Szczeniak \& Kahn, 1984). For instance, when biting into a baguette, one may perceive contrasting textures from the hard exterior to the soft interior, or a high energy to low energy texture change. However, after bolus formation one may perceive both soft and moist textures concurrently, both of which require a low level of oral processing energy.

The current study was designed to look at cross-cultural differences of liked and disliked textures among three different populations: 1) North American (U.S.A, N = 124), 2) European (Poland, $\mathrm{N}=73$ ) and Asian (Singapore, $\mathrm{N}=91$ ). The survey sites were chosen to provide a broad perspective towards texture contrasts across three highly populated continents. Individuals from each population were asked to rate the importance of texture and foods having multiple textures. They were then asked to describe textures for their most liked and disliked foods. An online survey was used to collect responses and a coding schema based on an established texture classification system was used to analyze results. Building on previous work, we anticipate universal liking for texture and multiple textures within food; however, cultural shifts in types of textures mentioned for liked and disliked foods are expected.

\section{Methods and Materials}

\section{Participants}


A total of 288 individuals with an age ranging from 18 to 77 years old $[$ mean $(M)=30.24$, standard deviation $(\mathrm{SD})=11.91$ ] participated in the study. Participants in the U.S.A. and Poland were recruited from the general population, while Singaporean participants were recruited from a student population. Thus, representing a European, Asian, and North American population, respectively (see Table 2). All participants provided written informed consent and were compensated for their participation. The study was conducted according to the Declaration of Helsinki and approved by the University of Tennessee IRB review for research involving human subjects (IRB \# 17-03973-XP). (Participants required approval: Texture Survey Consent.pdf)

Table 2. Descriptive statistics for age, gender, height, and weight of each sample by country.

\begin{tabular}{|c|c|c|c|c|c|c|c|}
\hline Sex & Country & & $N$ & Min & Max & Mean & SD \\
\hline \multirow[t]{8}{*}{ Men } & \multirow[t]{2}{*}{ All Men } & Age & \multirow{2}{*}{125} & 18.0 & 70.0 & 28.5 & 10.4 \\
\hline & & $\mathrm{BMI}$ & & 17.3 & 46.9 & 23.9 & 5.0 \\
\hline & \multirow[t]{2}{*}{ Poland } & Age & \multirow{2}{*}{50} & 18.0 & 57.0 & 28.2 & 8.2 \\
\hline & & $\mathrm{BMI}$ & & 17.3 & 38.3 & 21.9 & 3.7 \\
\hline & \multirow[t]{2}{*}{ Singapore } & Age & \multirow{2}{*}{40} & 21.0 & 30.0 & 23.8 & 1.9 \\
\hline & & $\mathrm{BMI}$ & & 18.6 & 30.0 & 22.7 & 2.5 \\
\hline & \multirow[t]{2}{*}{ U.S.A. } & Age & \multirow{2}{*}{35} & 19.0 & 70.0 & 34.3 & 15.2 \\
\hline & & $\mathrm{BMI}$ & & 17.8 & 46.9 & 28.3 & 6.0 \\
\hline \multirow[t]{8}{*}{ Woman } & \multirow[t]{2}{*}{ All Women } & Age & \multirow{2}{*}{163} & 18.0 & 77.0 & 31.4 & 12.7 \\
\hline & & $\mathrm{BMI}$ & & 18.2 & 46.8 & 24.7 & 6.8 \\
\hline & \multirow[t]{2}{*}{ Poland } & Age & \multirow{2}{*}{23} & 18.0 & 52.0 & 28.4 & 8.4 \\
\hline & & $\mathrm{BMI}$ & & 18.7 & 31.6 & 25.3 & 3.4 \\
\hline & \multirow[t]{2}{*}{ Singapore } & Age & \multirow{2}{*}{51} & 21.0 & 26.0 & 21.8 & 1.1 \\
\hline & & $\mathrm{BMI}$ & & 12.3 & 26.7 & 20.4 & 2.6 \\
\hline & \multirow[t]{2}{*}{ U.S.A. } & Age & \multirow{2}{*}{89} & 20.0 & 77.0 & 37.7 & 13.4 \\
\hline & & $\mathrm{BMI}$ & & 18.2 & 46.8 & 27.0 & 7.9 \\
\hline
\end{tabular}

\section{Survey}

A separate online survey was deployed for each participating population. The survey was presented in English for Singapore and U.S.A., while the Polish authors translated and presented their questions in Polish. The survey began with a general definition for texture [adapted from (Szczesniak, 2002)] 
Texture is the sensation from the structural, mechanical, and surface properties of the foods detected through the senses of vision, hearing, and touch. In other words, texture is the way food and drink feels in your mouth.

Next, participants were asked to use a 7-point category scale to assess 1) texture's importance to food liking ("Not Important at All" to "Extremely Important") and 2) level of agreement to the enjoyment of multiple textures ("Extremely Disagree" to "Extremely Agree").

1. In general, how important is texture to your liking of a food?

2. In order for a food to be enjoyable, it has to have multiple textures.

After ratings, the participants were asked to specify their most liked and disliked food or dish. They were then asked to list 3 texture qualities that make them like or dislike the food or dish. The supplemental text boxes were not mandatory in case the food/dish did not have three liked or disliked textures. Additionally, the participants were asked for their gender, age, nationality, native language, height and weight.

\section{Statistical Analysis}

Only individuals claiming nationality that matched the corresponding population of interest was analyzed. Analysis was completed in SAS 9.4 (SAS Institute Inc., Cary, NC). The rating of texture importance and level of agreement to the enjoyment of multiple textures were analyzed through a one-way ANCOVA with population as the predictive factor. Age and BMI were used as cofactors in these models.

The frequency of common liked and disliked texture terms were quantified for each population and the frequency of those popular terms ( $>5$ mentions) was compared. A Fisher's exact test was used and post-hoc comparisons among populations were Bonferroni corrected. 
Significant terms that may be due to language (especially Polish) were verified by a native Polish speaking author.

Texture quality terms were categorized into an established classification system for texture types (Guinard \& Mazzucchelli, 1996; Szczeniak, 1963) (Table 1). Specifically, the class of texture characteristic (mechanical, geometric and other) and the subsequent primary and secondary characteristics were recorded for each texture term. Next, three texture variables were calculated from the coded categories: texture contrast, texture classes, and texture combinations. A texture contrast was defined as a food being described by an active and passive texture (e.g. hard/crispy and soft/moist). This was a binary variable - either there was or there was not a contrast between two or more textures for a particular food. Texture combinations represented the number of different primary or secondary characteristics for a particular food. Texture classes represented the number of different classes of texture characteristics, providing a measure of the diversity in texture terms used. Texture contrasts and combinations have been described in previous literature (Szczeniak \& Kahn, 1984). For texture class and combination variables, a mixed effects model was used in which liked and disliked terms were used as the within variables and population as the between participants variable. A binomial mixed regression model was used to measure differences within liked foods and among populations. For all three models, age and BMI were set as covariates.

\section{Results}

There were no differences in the importance of texture to food liking among populations $\left(\mathrm{F}_{2,269}=\right.$ 1.62, $p=0.20, d=0.17$, with each rating food texture relatively important for liking: U.S.A.

$($ Mean \pm standard deviation $=5.14 \pm 1.51)$, Poland $(5.12 \pm 1.31)$ and Singapore $(5.35 \pm 1.25)$ However, the U.S.A. population $(3.42 \pm 1.53)$ put less importance on foods having multiple 
textures in order to be enjoyed compared to Polish (4.26 \pm 1.75$)$ and Singapore $(4.39 \pm 1.32)$

populations $\left(\mathrm{F}_{2,273}=10.12, p<0.001, d=0.53\right)$.

Table 3. List of popular texture terms used for liked and disliked foods. Terms in the same row that do not share a letter are significantly different from each other $(p>0.05)$

Liked Foods

\begin{tabular}{|c|c|c|c|c|c|c|c|}
\hline Terms & Polish & Singapore & U.S.A. & Terms & Polish & Singapore & U.S.A. \\
\hline chewy & $1.88 \% \mathrm{~b}$ & $9.35 \% \mathrm{a}$ & $7.24 \% \mathrm{a}$ & chewy & $6.85 \%$ & $4.00 \%$ & $\begin{array}{l}4.10 \% \\
1.87 \%\end{array}$ \\
\hline creamy & $1.88 \% \mathrm{~b}$ & $3.27 \% \mathrm{ab}$ & $7.24 \% \mathrm{a}$ & crunchy & $0 \% \mathrm{~b}$ & $5.71 \%$ a & $a b$ \\
\hline crispy & $13.75 \%$ & $7.01 \%$ & $8.62 \%$ & dry & $4.79 \%$ & $3.43 \%$ & $2.61 \%$ \\
\hline crunchy & $0.63 \% \mathrm{c}$ & $7.01 \% \mathrm{~b}$ & $18.62 \% \mathrm{a}$ & fatty & $4.11 \% \mathrm{a}$ & $0 \% \mathrm{~b}$ & $0.37 \% \mathrm{~b}$ \\
\hline elastic & $4.38 \% \mathrm{a}$ & $0 \% \mathrm{~b}$ & $0 \% \mathrm{~b}$ & grainy & $0.68 \%$ & $0.57 \%$ & $4.10 \%$ \\
\hline firm & $5.63 \% \mathrm{a}$ & $0.47 \% \mathrm{~b}$ & $4.14 \% \mathrm{a}$ & gritty & $0 \% \mathrm{~b}$ & $0 \% \mathrm{~b}$ & $3.73 \% \mathrm{a}$ \\
\hline hard & $0.00 \%$ & $1.87 \%$ & $2.41 \%$ & hard & $0 \% \mathrm{~b}$ & $13.14 \% \mathrm{a}$ & $1.87 \% \mathrm{~b}$ \\
\hline juicy & $1.25 \%$ & $3.27 \%$ & $2.07 \%$ & lumpy & $2.74 \%$ & $1.14 \%$ & $\begin{array}{c}1.87 \% \\
13.06 \%\end{array}$ \\
\hline layered & $3.13 \% \mathrm{a}$ & $0 \% \mathrm{~b}$ & $0.34 \% \mathrm{ab}$ & mushy & $2.74 \% \mathrm{a}$ & $8 \%$ ab & $\mathrm{a}$ \\
\hline melty & $2.50 \%$ & $0.93 \%$ & $2.41 \%$ & rough & $0.68 \% \mathrm{~b}$ & $8 \%$ a & $1.49 \% \mathrm{~b}$ \\
\hline moist & $4.38 \%$ & $3.27 \%$ & $2.41 \%$ & rubbery & $2.74 \%$ & $1.14 \%$ & $\begin{array}{c}1.87 \% \\
12.31 \%\end{array}$ \\
\hline rough & $0.63 \% \mathrm{ab}$ & $4.67 \%$ a & $0.69 \%$ b & slimy & $0 \% \mathrm{~b}$ & $9.14 \% \mathrm{a}$ & $\mathrm{a}$ \\
\hline smooth & $5.63 \% \mathrm{~b}$ & $16.36 \% \mathrm{a}$ & $9.66 \% \mathrm{ab}$ & slippery & $5.48 \%$ & $1.14 \%$ & $1.49 \%$ \\
\hline soft & $18.75 \%$ & $19.16 \%$ & $13.10 \%$ & soft & $4.79 \%$ & $8.57 \%$ & $3.73 \%$ \\
\hline springy & $4.38 \% \mathrm{a}$ & $0.47 \% \mathrm{ab}$ & $0 \% \mathrm{~b}$ & squishy & $2.74 \%$ & $2.86 \%$ & $3.73 \%$ \\
\hline tender & $6.88 \%$ & $5.14 \%$ & $5.52 \%$ & sticky & $10.27 \% \mathrm{a}$ & $4 \% a b$ & $\begin{array}{c}2.24 \% \mathrm{~b} \\
2.99 \%\end{array}$ \\
\hline- & - & - & - & stringy & $6.85 \% \mathrm{a}$ & $0 \% \mathrm{~b}$ & $a b$ \\
\hline- & - & - & - & tough & $10.27 \%$ & $3.43 \%$ & $4.48 \%$ \\
\hline- & - & - & - & watery & $4.79 \%$ & $2.86 \%$ & $1.87 \%$ \\
\hline
\end{tabular}

Differences among popular terms (> 5 mentions) across populations within liking are marked on Table 3 (for a list frequency for all terms, see Supp. Table 1. The top 5 texture words for each population's liked and disliked foods are shown in Table 4. Soft was widely listed when describing liked foods and was either the $1^{\text {st }}$ or $2^{\text {nd }}$ most popular descriptor for liked foods across all populations. Smooth, crispy, and crunchy were also commonly used to describe liked foods. Across populations there was less similarity in the terms to describe disliked foods. 


\begin{tabular}{llllll}
\hline & \multicolumn{2}{c}{ Liked } & \multicolumn{3}{c}{ Disliked } \\
\hline Poland & Singapore & U.S.A. & Poland & Singapore & U.S.A. \\
\hline Soft & Soft & Crunchy & Sticky & Hard & Mushy \\
Crispy & Smooth & Soft & Tough & Slimy & Slimy \\
Tender & Chewy & Smooth & Stringy & Soft & Tough \\
Firm & Crispy & Chewy & Chewy & Mushy & Chewy \\
Smooth & Crunchy & Creamy & Slippery & Rough & Grainy \\
\hline
\end{tabular}

Table 4. Across all cultures, 71 texture terms were mentioned for liked foods and 93 terms for disliked

A visualization of popular texture terms for liked and disliked foods are shown in Figure 1.

Populations used different popular terms for liked and disliked foods $(\chi 2=154.10, \mathrm{p}<0.001$ and $\chi 2=204.733, \mathrm{p}<0.001$, respectively) foods.

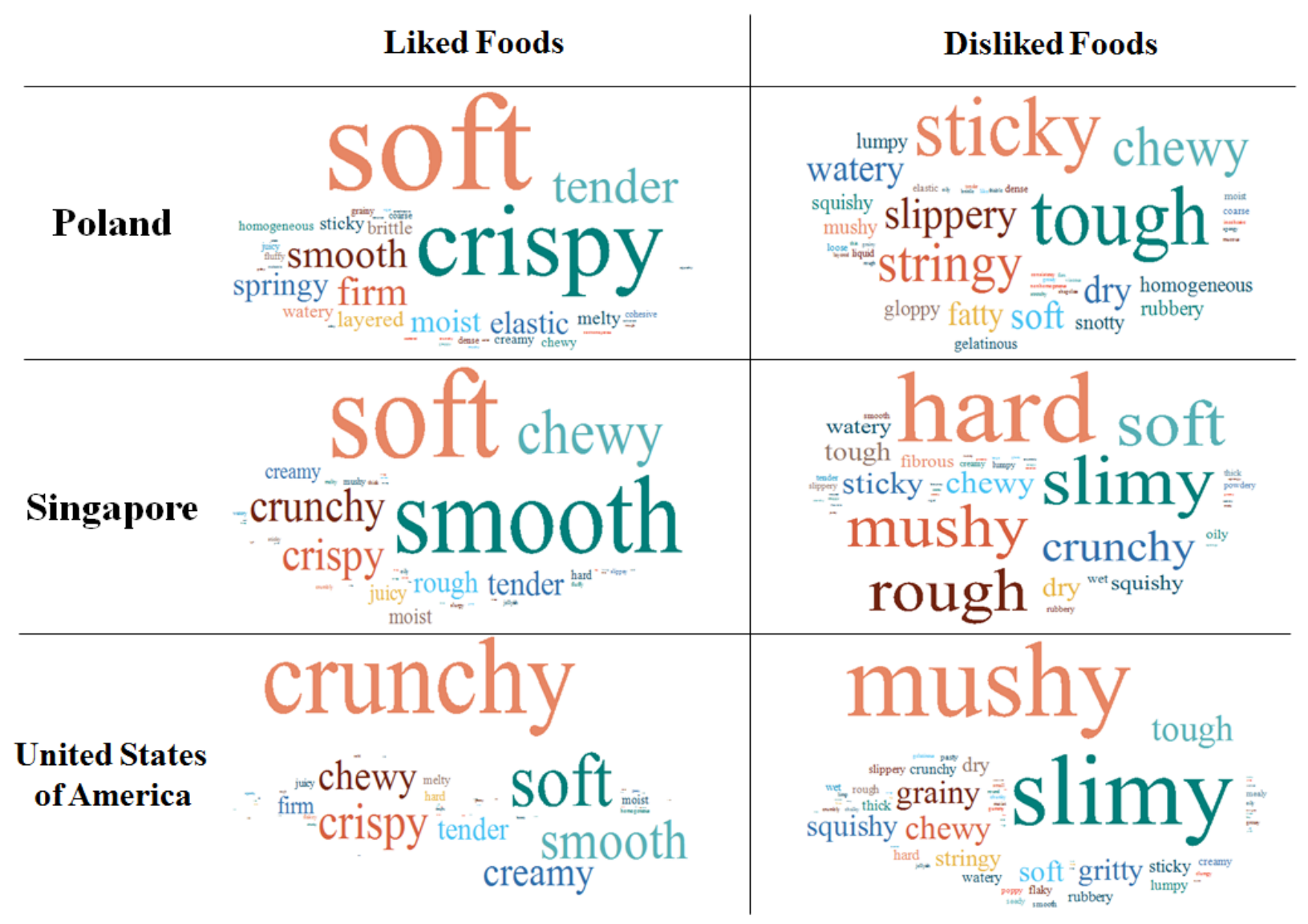


Figure 1. Word clouds of popular texture terms between food likings and among populations. Larger words represent a greater frequency of use within a population for liked or disliked foods.

As shown in Figure 2, more texture combinations and texture contrasts were mentioned for liked foods compared to disliked foods $\left(\mathrm{F}_{1,271}=12.73, \mathrm{p}<0.001, d=0.26\right.$ and $\mathrm{F}_{1,419}=63.01$, $\mathrm{p}<0.001, \mathrm{OR}=2.68, d=0.55$ respectively $)$, and this was independent of culture $\left(\mathrm{F}_{2,269}=2.05, \mathrm{p}\right.$ $=0.13$ and $\mathrm{F}_{2,419}=2.56, \mathrm{p}=0.08$ respectively).

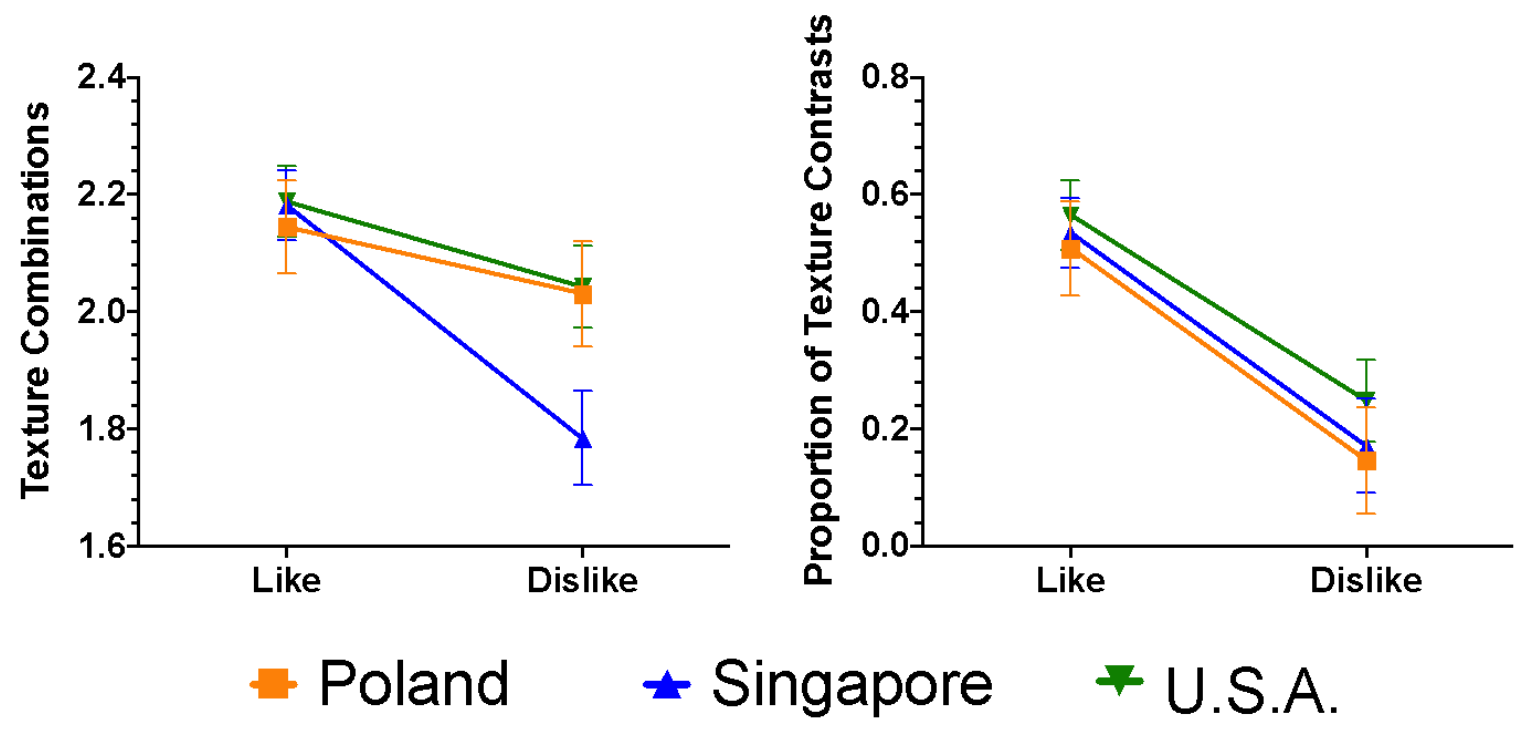

Figure 2. Frequency of texture contrasts and combinations mentioned for liked and disliked foods among populations.

However, an interaction between population and food liking was observed for texture characteristics $\left(\mathrm{F}_{2,271}=7.56, \mathrm{p}<0.001, d=0.38\right.$; Figure 3$)$. The Singapore population mentioned more texture characteristics for liked compared to disliked foods while the opposite trend was shown for U.S.A. and Polish populations. There were no other significant effects $(\mathrm{p}>0.05)$. 


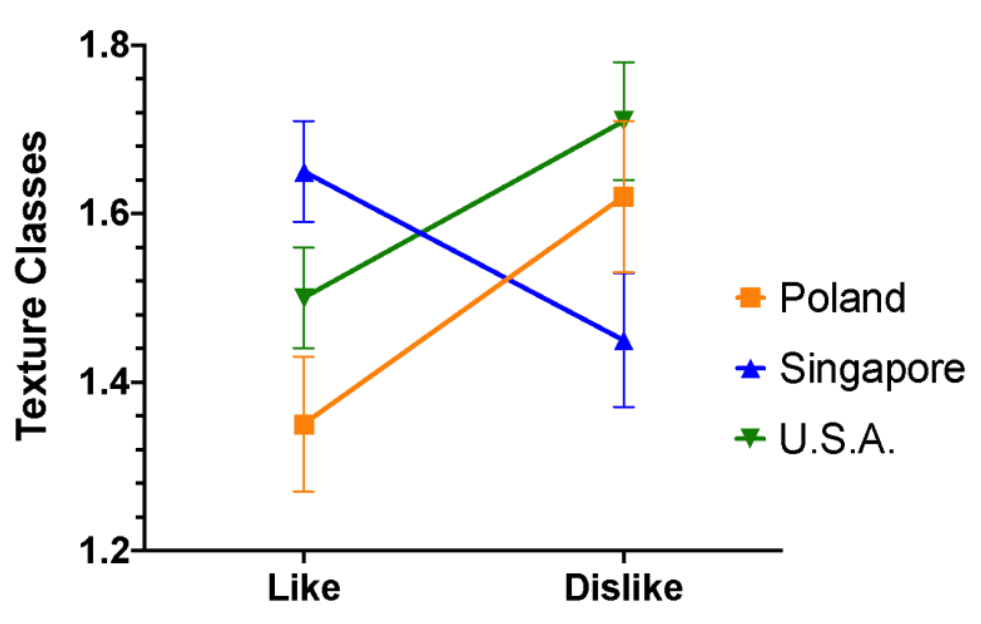

Figure 3. Frequency of texture characteristics mentioned for liked and disliked foods among populations. An interaction among population and food liking exists $(\mathrm{p}<0.001)$. From liked to disliked foods, the Asian population decreased their mention of texture characteristics while an increase was noticed for Poland and U.S.A.

\section{Discussion}

The classification of texture may fall under its main class of characteristics and subsequent primary and secondary properties as defined by Szczesniak (1963). In this study, we examined these notions and defining mechanisms across populations to consider the consistency and cultural-specificity of consumer attitudes towards texture contrasts and combinations across 3 distinct cultures.

As expected, texture in foods are important with all populations in the study reporting similarly high levels of importance. Texture is universally recognized as integral to making foods enjoyable to eat. Two similarly designed surveys asking individuals to openly report food attributes that contribute to the liking of several (30+) types of foods showed texture as the most frequently mentioned attribute followed by flavor (Luckett \& Seo, 2015; Szczesniak \& Kahn, 1971). Given the dynamic nature of texture changes during consumption, individual texture attributes are often not perceived in isolation, but accompanied by other textures. The importance of multiple textures to food appreciation was confirmed across the populations in the current 
study. In particular, the North American (U.S.A.) population rated the importance for foods having multiple textures to be less important for food pleasantness than the other two populations, even when controlling for age and BMI. The authors suggest two non-exclusive explanations for these findings: 1) the lack of awareness and/or 2) the abundance of processed foods typical in this population's diet. Processed foods often tend to be softer and with more uniform textures than less processed foods, and this can be attributed to increased fat and sugar as well as processing removing innate structures in the original foods (Campbell, Wagoner, \& Foegeding, 2017; de Graaf, 2012). For instance, an apple in its natural form is heterogeneously shaped with a hard exterior and juicy interior while its processed form, such as applesauce, has a flow of consistent particle size that makes it smooth. A higher familiarity and consumption of processed foods (Steele et al., 2016) may lead to less attention being given to texture heterogeneity and a lowering of its perceived importance.

The presence of multiple textures happens during initial evaluation and during mastication as the texture typically shifts from high to low energy. Thus, at any stage, there may be different combinations of textures and they may or may not be contrasting. We show that all individuals, irrelevant of culture, report more combinations and contrasts in liked foods/dishes compared to disliked foods/dishes. Therefore, although the U.S.A. population were found to place less importance on foods having multiple textures, these textures were still present among the most palatable foods. Similarly, Szczesniak and Kahn (1984) were the first to point out the importance of texture contrasts and combinations for a US population stating the "polarity between stimulating/energetic (i.e., firm, crisp) and passive/soothing (i.e., soft, creamy) texture characteristics form the foundation of many highly desirable texture combinations" (Szczeniak \& Kahn, 1984). In this paper, they describe four scenarios incorporating texture combinations and 
contrasts to increase enjoyment: 1) within a meal, 2) on the plate, 3) within a multiphase food, and 4) within a uniphase food during consumption. Whereas within meal texture combinations may demonstrate low to high energy shifts in texture (e.g. soup to meat), most answers to our survey dealt with contrasts of varying combinations for the multiphase or uniphase foods (e.g. steak being crispy then tender or smooth).

The textures within combinations and contrasts were not the same across cultures with many different texture terms being reported for liked and disliked foods (Figure 1). Past studies have examined texture term usage across populations with differences being demonstrated between and within cultures, consumers and experts (Blancher et al., 2007; Cardello et al., 1982; Drake, 1989; Lawless et al., 1997; Nishinari et al., 2008). For instance, it has been shown that English speakers use far less texture terms ( 70) (Drake, 1989; Szczesniak \& Kleyn, 1963) than those from Japan ( 400) (Hayakawa et al., 2005; Yoshikawa, Nishimaru, Tashiro, \& Yoshida, 1970), as the Japanese language includes many more onomatopoeic words for descriptions (e.g. fizz, crackle) (Hayakawa, Hatae, \& Shimada, 2000). In our study, we focused on collecting an unrestrained consumer lexicon for liked and dislike foods. Interestingly, collapsing across cultures, 22 more disliked texture terms were mentioned than liked terms (Supp. Table 1) - a finding supporting the view that texture is often more salient when unpleasant (Szczesniak, 2002). Additionally, it has been stated that while flavor drives food liking, texture is often the food attribute responsible for food rejection (Cardello, 1996).

One cross-cultural study, similar to ours, categorized each term by its main class of characteristics and showed that cultures similarly use more mechanical and geometrical terms (Nishinari et al., 2008). This notion may be true for our study as well; however, we asked a different question, "how does the number of main class of texture characteristics within a liked 
and disliked food combination culturally differ?" Here, we found differences across populations. The Asian (Singapore) population showed more main classes of characteristics within a liked than a disliked combination of textures. The opposite trend was found in the European (Polish) and North American populations. In this regard foods that are too diverse in their textures tended to be aversive for the two Western cultures, but were reported as more palatable for the Asian population in our survey. This may reflect differences in the breadth of texture awareness across the three different cultures and future studies are needed to describe these differences in more detail and across a wider geographical population. It is important to note, however, that Singapore has a very diverse population in close proximity to distinct styles of cuisine across east Asia including influence from western cultures. Therefore, individuals in this environment are accustomed to many types of foods with varying texture.

\section{Limitations}

This study provides the consumer attitudes towards texture across 3 unique populations, however the authors made some methodological concessions to complete this study. The study was distributed online, meaning no actual food was consumed and the participants were not strictly controlled in the construction of their responses. Additionally, the Singaporean population were university students. While the authors controlled for age in the statistical analyses, other sociocultural factors could be unique to the student population. Lastly, the Polish population was administered the survey in Polish, while the other populations completed the survey in English. There is a possibility that the available vocabulary for texture descriptors is different across the languages (i.e. Polish and English) used in this study.

\section{Conclusion}


This study highlights that texture combinations and contrasts are an important factor for texture acceptance across three cross-cultural populations. The importance of texture combinations and contrasts was stable across populations, though diverse textures were associated with greater palatability in Asia and having multiple textures was found to be less important for food liking in the other two populations.

Acknowledgements: The authors would like to thank Lang Li for assistance with survey preparation and data collection.

Conflict of Interest: The authors declare no conflict of interest.

Author Contributions: Design (R.P, C.R.T.), acquisition of data (all authors), analysis (R.P. C.R.T), interpretation (all authors), drafting/revising manuscript (all authors). 


\section{References}

Blancher, G., Chollet, S., Kesteloot, R., Hoang, D. N., Cuvelier, G., \& Sieffermann, J.-M. (2007). French and Vietnamese: How do they describe texture characteristics of the same food? A case study with jellies. Food Quality and Preference, 18(3), 560-575. https://doi.org/10.1016/J.FOODQUAL.2006.07.006

Campbell, C. L., Wagoner, T. B., \& Foegeding, E. A. (2017). Designing foods for satiety: The roles of food structure and oral processing in satiation and satiety. Food Structure, 13, 1-12. https://doi.org/10.1016/J.FOOSTR.2016.08.002

Cardello, A. V., Maller, O., Kapsalis, J. G., Segars, R. A., Sawyer, F. M., Murphy, C., \& Moskowitz, H. R. (1982). Perception of Texture by Trained and Consumer Panelists. Journal of Food Science, 47(4), 1186-1197. https://doi.org/10.1111/j.13652621.1982.tb07646.x

Cardello, A. V. (1996). The role of the human senses in food behavior. In Texture. Cereal foods world (USA) (p. II). Retrieved from http://agris.fao.org/agrissearch/search.do?recordID=US9620352

de Graaf, C. (2012). Texture and satiation: The role of oro-sensory exposure time. Physiology \& Behavior, 107(4), 496-501. https://doi.org/10.1016/J.PHYSBEH.2012.05.008

Drake, B. (1989). Sensory Textural/Rheological Properties?A Polyglot List. Journal of Texture Studies, 20(1), 1-27. https://doi.org/10.1111/j.1745-4603.1989.tb00417.x

Guinard, J. X., \& Mazzucchelli, R. (1996). The sensory perception of texture and mouthfeel. Trends in Food Science and Technology, 7(7), 213-219. https://doi.org/10.1016/09242244(96)10025-X

Hayakawa, F., Hatae, K., \& Shimada, J. (2000). Characterization of onomatopoeia and mimetic languages of monthfeel. The Japanese Society for Food Science and Technology, 47(3), 197-207.

Hayakawa, F., Ioku, K., Akuzawa, S., Saito, M., Nishinari, K., Yamano, Y., \& Kohyama, K. (2005). Collection of Japanese texture terms (Studies on Japanese texture terms Part 1). The Japanese Society for Food Science and Technology, 52(8), 376-346.

Hyde, R. J., \& Witherly, S. A. (1993). Dynamic contrast: A sensory contribution to palatability. Appetite. https://doi.org/10.1006/appe.1993.1032

Lawless, H., Vanne, M., \& Tuorila, H. (1997). Categorization of English and Finnish Texture Terms Among Consumers and Food Professionals. Journal of Texture Studies, 28(6), 687708. https://doi.org/10.1111/j.1745-4603.1997.tb00147.x

Luckett, C. R., \& Seo, H.-S. (2015). Consumer Attitudes Toward Texture and Other Food Attributes. Journal of Texture Studies, 46(1), 46-57. https://doi.org/10.1111/jtxs.12110

Nishinari, K., Hayakawa, F., Xia, C., Huang, L., Meullenet, J., \& Sieffermann, J. (2008). Comparative Study Of Texture Terms: English, French, Japanese And Chinese. Journal of Texture Studies, 39(5), 530-568. https://doi.org/10.1111/j.1745-4603.2008.00157.x

Rohm, H. (1990). Consumer Awareness Of Food Texture In Austria. Journal of Texture Studies, 21(3), 363-374. https://doi.org/10.1111/j.1745-4603.1990.tb00485.x

Steele, E. M., Baraldi, L. G., Louzada, M. L. da C., Moubarac, J.-C., Mozaffarian, D., \& Monteiro, C. A. (2016). Ultra-processed foods and added sugars in the US diet: evidence from a nationally representative cross-sectional study. BMJ Open, 6(3), e009892. https://doi.org/10.1136/BMJOPEN-2015-009892 
Szczeniak, A. ., \& Kahn, E. L. (1984). Texture Contrasts and Combinations: a Valued Consumer Attribute. Journal of Texture Studies, 15(3), 285-301. https://doi.org/10.1111/j.17454603.1984.tb00385.x

Szczeniak, A. S. (1963). Classification of Textural Characteristics. Journal of Food Science, 28(4), 385-389. https://doi.org/10.1111/j.1365-2621.1963.tb00215.x

Szczesniak, A. S. (1971). Consumer Awareness Of Texture And Of Other Food Attributes, II. Journal of Texture Studies, 2(2), 196-206. https://doi.org/10.1111/j.17454603.1971.tb00581.x

Szczesniak, A. S. (1972). Consumer awareness of and attitudes to food texture ii. Children and teenagers. Journal of Texture Studies, 3(2), 206-217. https://doi.org/10.1111/j.17454603.1972.tb00624.x

Szczesniak, A. S. (2002). Texture is a sensory property, 13, 215-225.

Szczesniak, A. S., \& Kahn, E. L. (1971). Consumer Awareness Of And Attitudes To Food Texture. Journal of Texture Studies, 2(3), 280-295. https://doi.org/10.1111/j.17454603.1971.tb01005.x

Szczesniak, A. S., \& Kleyn, D. H. (1963). Consumer awareness of texture and of other food attributes. Food Technology, 17, 74-77.

Yoshikawa, S., Nishimaru, S., Tashiro, T., \& Yoshida, M. (1970). Collection and classification of words for description of food texture: Collection of words. Journal of Texture Studies, 1 , 437-442. 
Supplementary Tables

Table 1. Descriptive texture terms used for liked and disliked foods among populations.

\begin{tabular}{|c|c|c|c|}
\hline & Liked & oods & \\
\hline Terms & Polish (A) & Sirgapore (B) & LSA $(C)$ \\
\hline baunay & $0.00 \% 6$ & $0.47 \%$ & $0.00 \%$ \\
\hline brittle & $2.50 \% 6$ & $0.00 \% 6$ & $0.34 \%$ \\
\hline butteny & $0.00 \% 6$ & $0.00 \% 6$ & 0.6996 \\
\hline hew & $1.89 \%$ & $9.39 \% 6$ & $7.24 \% 6$ \\
\hline tiunky & $0.00 \%$ & 0.476 & $0.69 \%$ \\
\hline Darse & $1.25 \%$ & $0.00 \%$ & $0.00 \% 6$ \\
\hline wherent & $0.63 \%$ & $0.00 \%$ & $0.00 \%$ \\
\hline Dhesive & $1.25 \%$ & 0.476 & $0.00 \%$ \\
\hline gurse & $0.69 \%$ & $0.00 \%$ & $0.00 \%$ \\
\hline geamy & $1.88 \%$ & $3.2 \pi 6$ & $7.24 \%$ \\
\hline Gispy & 13. $\pi 56$ & $7.01 \%$ & $8.62 \%$ \\
\hline Gumbly & $0.00 \%$ & 0.9376 & $0.00 \%$ \\
\hline Gundiv & $0.63 \%$ & $7.01 \%$ & $18.62 \%$ \\
\hline Gusty & $0.00 \%$ & $0.4 \pi 6$ & $0.34 \%$ \\
\hline derse & $1.25 \%$ & $0.00 \% 6$ & $0.34 \%$ \\
\hline daughy & $0.00 \% 6$ & $0.00 \% 6$ & 0.6996 \\
\hline dry & $0.00 \%$ & $0.00 \%$ & $0.3 \% \%$ \\
\hline elatic & 4.3396 & $0.00 \% 6$ & $0.00 \%$ \\
\hline firm & $5.67 \%$ & $0.47 \%$ & $4.14 \%$ \\
\hline flakey & $0.00 \% 6$ & $0.00 \% 6$ & $1.03 \%$ \\
\hline flexible & $0.00 \%$ & $0.00 \%$ & $0.3 \% \%$ \\
\hline fluffy & $1.25 \%$ & 0.9376 & $0.34 \% 6$ \\
\hline glappN & $0.67 \%$ & $0.00 \% 6$ & $0.00 \%$ \\
\hline goney & $0.00 \%$ & $0.4 \pi 6$ & 0.6996 \\
\hline grainy & $1.25 \%$ & $0.4 \pi 6$ & $0.00 \%$ \\
\hline granular & $0.00 \%$ & $0.00 \% 6$ & $0.3 \% \%$ \\
\hline greasy & $0.00 \% 6$ & $0.00 \% 6$ & 0.6996 \\
\hline gritty & $0.00 \%$ & $0.00 \% 6$ & $0.34 \%$ \\
\hline hard & $0.00 \% 6$ & 1.876 & $2.41 \%$ \\
\hline hompgeneous & $1.89 \%$ & $0.00 \%$ & $1.03 \%$ \\
\hline in ares ive & $0.63 \%$ & $0.00 \% 6$ & $0.00 \%$ \\
\hline iellyish & $0.00 \% 6$ & 0.9376 & $0.00 \%$ \\
\hline juig & $1.25 \%$ & $3.27 \%$ & 2.076 \\
\hline laof & $0.00 \% 6$ & $0.4 \pi \%$ & $0.00 \% 6$ \\
\hline layered & $3.13 \%$ & $0.00 \%$ & $0.34 \%$ \\
\hline light & $0.00 \%$ & $0.4 \pi 6$ & $0.3 \% \%$ \\
\hline liquid & $0.00 \%$ & $0.00 \%$ & $0.34 \%$ \\
\hline malleable & $0.63 \%$ & $0.4 \pi / 6$ & $0.00 \% 6$ \\
\hline mettr & $2.50 \% 6$ & 0.9376 & $2.41 \%$ \\
\hline mast & 4.3396 & $3.2 \pi 6$ & 2.4196 \\
\hline mushy & $0.63 \% 6$ & $1.40 \% 6$ & $0.34 \%$ \\
\hline nanhamagenaus & $0.67 \% 6$ & $0.00 \% 6$ & $0.00 \%$ \\
\hline aily & $0.00 \% 6$ & 0.9376 & $0.34 \%$ \\
\hline ouze & $0.00 \%$ & $0.00 \%$ & $0.34 \%$ \\
\hline pithy & $0.63 \% 6$ & $0.00 \% 6$ & $0.00 \% 6$ \\
\hline rigid & $0.63 \%$ & $0.00 \% 6$ & $0.00 \%$ \\
\hline rough & $0.63 \% 6$ & $4.6 \% 6$ & 0.699 \\
\hline saucy & $0.00 \%$ & $0.4 \pi 6$ & $0.3 \% \%$ \\
\hline scattered & $0.63 \% 6$ & 0.0006 & $0.00 \% 6$ \\
\hline $\begin{array}{l}\text { shiny } \\
\text { shy }\end{array}$ & $0.00 \%$ & $0.47 \%$ & $0.00 \%$ \\
\hline silky & $0.63 \%$ & $0.4 \pi 6$ & $0.34 \%$ \\
\hline slick & $0.63 \%$ & $0.00 \%$ & $0.3 \% \%$ \\
\hline slimv & $0.00 \% 6$ & $0.00 \% 6$ & $0.34 \%$ \\
\hline slippery & $0.00 \% 6$ & 0.9376 & $0.00 \% 6$ \\
\hline slurpy & $0.00 \% 6$ & 0.9376 & $0.3 \% 6$ \\
\hline small & $0.00 \%$ & $0.4 \pi 6$ & $0.00 \%$ \\
\hline smaoth & $5.67 \%$ & $16.3 \oplus 6$ & $9.6 \% 76$ \\
\hline snappy & $0.00 \% 6$ & $0.47 \%$ & $0.34 \%$ \\
\hline soft & $18 . \pi 96$ & 19.1076 & $13.10 \% 6$ \\
\hline solid & $0.67 \%$ & $0.4 \pi / 6$ & 0.6996 \\
\hline springy & 4.3396 & $0.4 \pi 6$ & $0.00 \%$ \\
\hline squishy & $0.63 \%$ & $0.00 \%$ & $0.69 \%$ \\
\hline stidor & $2.50 \%$ & 0.9376 & $0.34 \%$ \\
\hline strirgy & $0.00 \% 6$ & $0.4 \pi / 6$ & $0.00 \% 6$ \\
\hline tender & $6.89 \%$ & $5.14 \%$ & $5.52 \%$ \\
\hline thick & $0.00 \% 6$ & 0.9376 & $0.34 \%$ \\
\hline thin & $0.00 \%$ & $0.00 \% 6$ & $0.3 \% \%$ \\
\hline tharny & $0.00 \% 6$ & $0.4 \pi / 6$ & $0.00 \%$ \\
\hline touthiness & $0.00 \% 6$ & $0.00 \% 6$ & $0.34 \%$ \\
\hline waten & $2.50 \% 6$ & 0.9376 & $0.34 \%$ \\
\hline wiggry & $0.00 \%$ & $0.00 \%$ & $0.3 \% \%$ \\
\hline 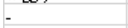 & - & - & - \\
\hline- & - & - & - \\
\hline - & - & - & - \\
\hline & - & - & - \\
\hline - & - & - & - \\
\hline - & - & - & - \\
\hline - & - & - & - \\
\hline - & - & - & - \\
\hline - & - & - & - \\
\hline - & - & - & - \\
\hline - & - & - & - \\
\hline - & - & - & - \\
\hline - & - & - & - \\
\hline - & - & - & - \\
\hline - & - & - & - \\
\hline- & - & - & - \\
\hline - & - & - & - \\
\hline - & - & - & - \\
\hline$=$ & - & - & - \\
\hline - & - & - & 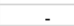 \\
\hline - & - & - & - \\
\hline & & - & - \\
\hline
\end{tabular}

